\title{
Scientific guidance to fight the pandemic: the Coronavirus Disease 2019 (COVID-19)
}

\author{
Zifeng Yang ${ }^{1}$, Zhiqi Zeng ${ }^{1}$, Ke Wang ${ }^{2}$, Nanshan Zhong ${ }^{1}$, Jianxing $\mathrm{He}^{1}$ \\ ${ }^{1}$ State Key Laboratory of Respiratory Disease, National Clinical Research Center for Respiratory Disease, National Center for Respiratory Medicine, \\ Guangzhou Institute of Respiratory Health, The First Affiliated Hospital of Guangzhou Medical University, Guangzhou, China; ${ }^{2}$ Hengqin \\ WhaleMed Technology Co., Ltd., Zhuhai, China \\ Correspondence to: Jianxing He, Nanshan Zhong. State Key Laboratory of Respiratory Disease, National Clinical Research Center for Respiratory \\ Disease, National Center for Respiratory Medicine, Guangzhou Institute of Respiratory Health, The First Affiliated Hospital of Guangzhou Medical \\ University, Guangzhou, China. Email: hejx@vip.163.com; nanshan@vip.163.com.
}

Submitted Jan 15, 2021. Accepted for publication Feb 10, 2021.

doi: $10.21037 /$ jtd-2021-02

View this article at: http://dx.doi.org/10.21037/jtd-2021-02

Forecasting System (public beta) is available online at bttp:// covid19. whalemed.cn, testing is welcome.

The Coronavirus Disease 2019 (COVID-19) Forecasting System (public beta) was developed by the National Respiratory Medicine Center of First Affiliated Hospital of Guangzhou Medical University, Guangzhou Institute of Respiratory Health, and National Clinical Research Center for Respiratory Disease and is an important part of the Respiratory Center Health Big Data Platform.

During the initial outbreak of severe acute respiratory syndrome coronavirus 2 (SARS-CoV-2), a core research team led by Academician Nanshan Zhong and Prof. Jianxing He collaborated with a third-party professional artificial intelligence team to develop a model to accurately predict the epidemic trend nationally in the country and in high-risk areas of China, and to provide information on professional intervention for reference (the model was published in 7 Thorac Dis 2020;12:165-174. http:// jtd.amegroups.com/article/view/36385/html). This local prediction model will provide strong scientific support in the fight against SARS-CoV-2, and its prediction accuracy is significantly better than that of foreign forecasting models.

Based on previous experience and monitoring of epidemics in numerous places around the world, the core research team collected open data on SARS-CoV-2 epidemics in different regions, and considered the impact of different measures, such as mask wearing, on the spread of the coronavirus. Through this, the "Coronavirus
Disease 2019 (COVID-19) Forecasting System (public beta)" (independent intellectual property rights ownership: Guangzhou Institute of Respiratory Health) was successfully developed to scientifically predict the trend of the pandemic around the world. This system has been made available online, and scientists around the world are welcome to test it and give the feedback.

The system includes the following functions: a world map, pandemic trends in each country, pandemic forecasting, vaccine progress and other functions.

World map (Figure 1): the map visually displays the latest information on the pandemic from various countries. Features include evaluation of the effective reproduction number Rt and a "mask on" index.

Pandemic trends in China: visual analysis of domestic pandemic data and maps showing China's overall trend (Figure 2), and provincial and municipal trends (Figure 3).

Pandemic trends in countries: visual analysis of past pandemic data of from every country in the world (Figure 4).

User experience: users can adjust the intervention time and intensity, population, transmission time, R0, and other influential epidemiological parameters according to their needs, and quickly obtain the results (Figure 5).

USA prediction: as of February 7, 2021, the cumulative number of confirmed diagnoses is estimated to be approximately 27 million (95\% CI: 28,934,888-24,991,037; Figure 6A).

Russia prediction: as of February 7, 2021, the 


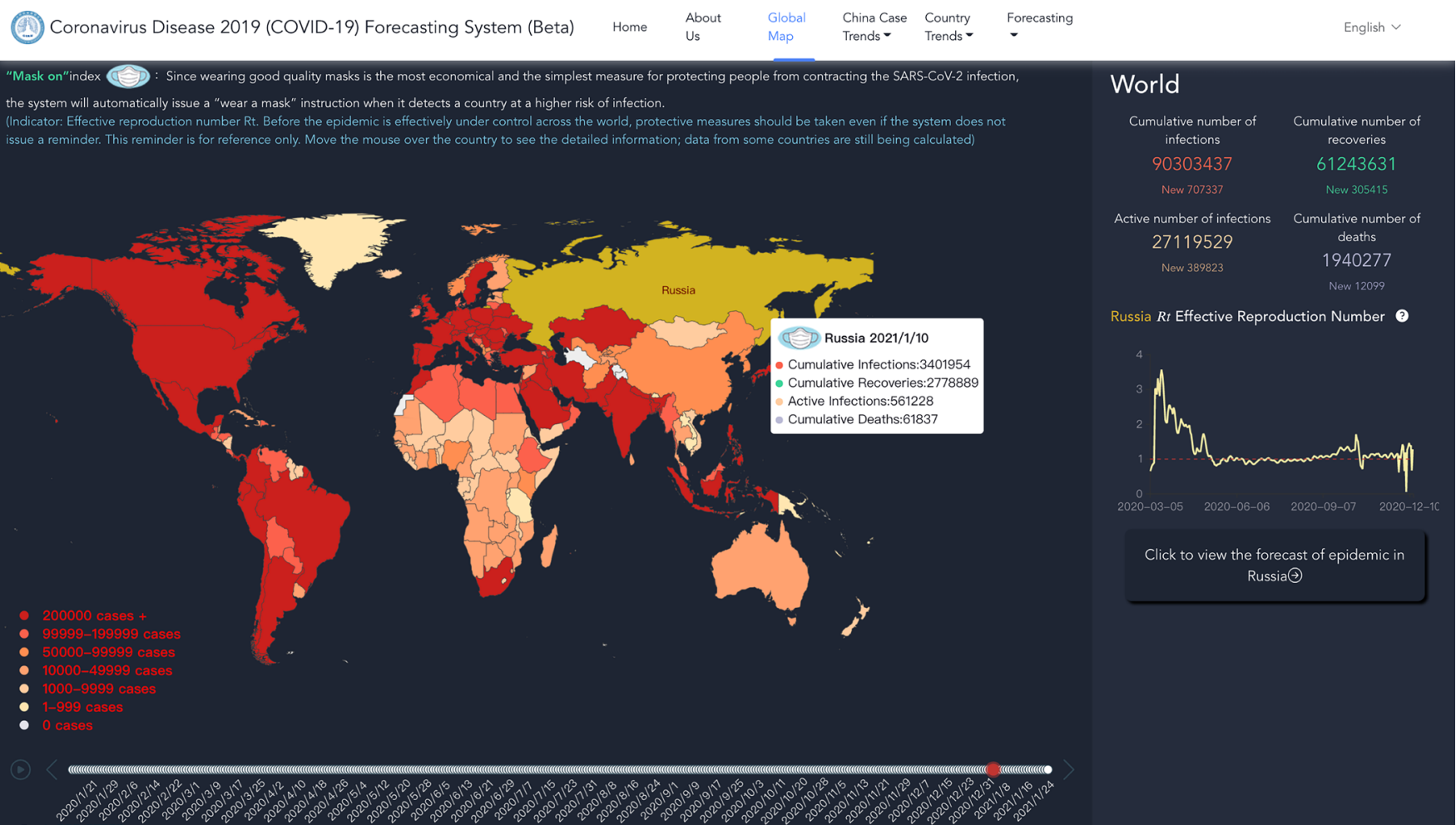

Figure 1 The SARS-CoV-2 pandemic map for the world.

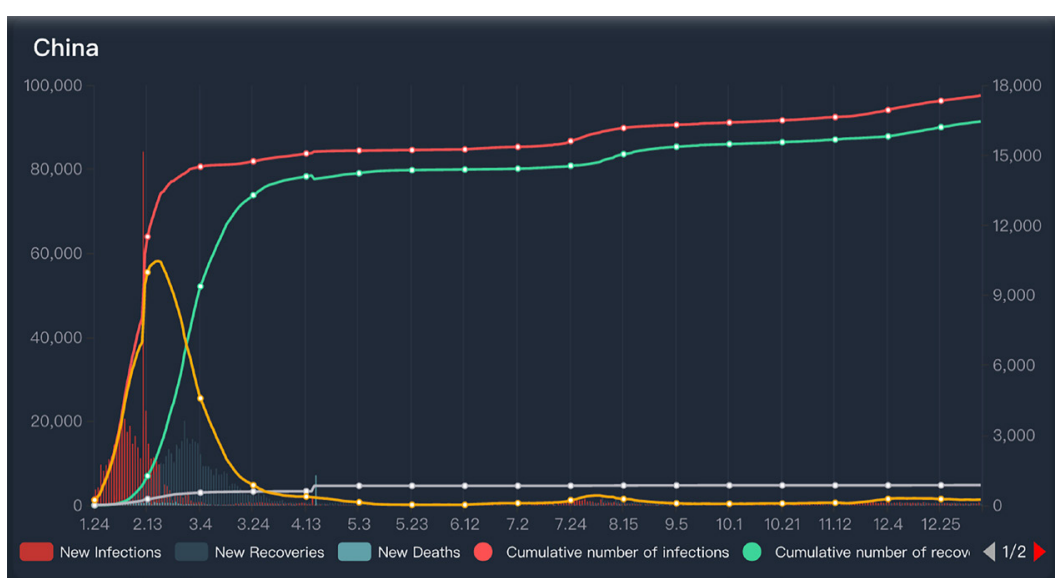

Figure 2 The pandemic information for China. Red bar, new infections; Black bar, new recoveries; Blue bar, new deaths; Red line, cumulative number of infections; Green line, cumulative number of recoveries; Gray line, cumulative number of deaths; Yellow line, active number of infections. 


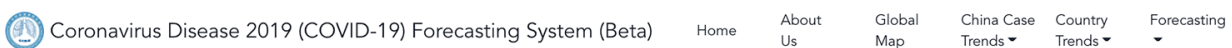

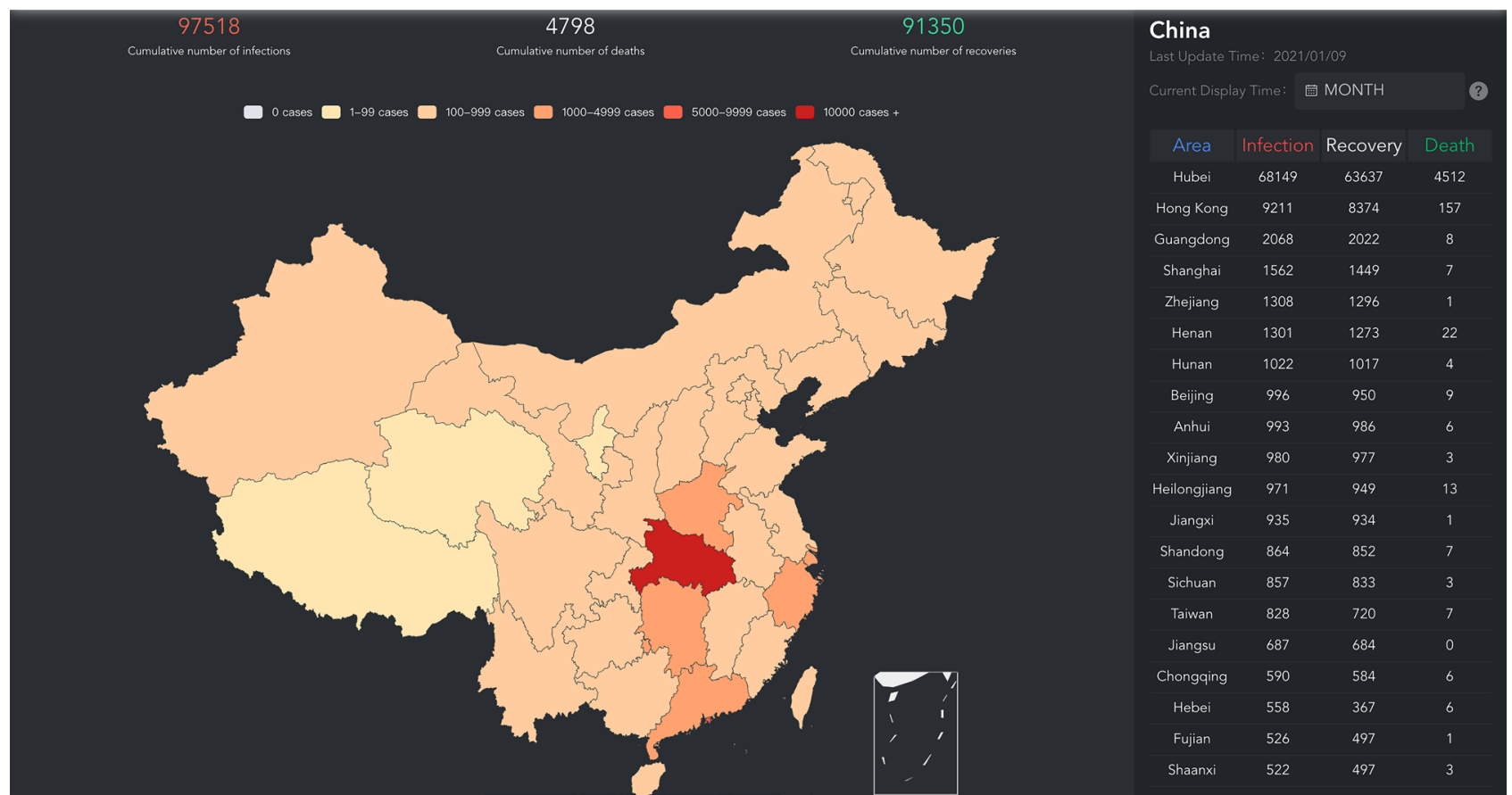

Figure 3 The pandemic data for different areas in China.

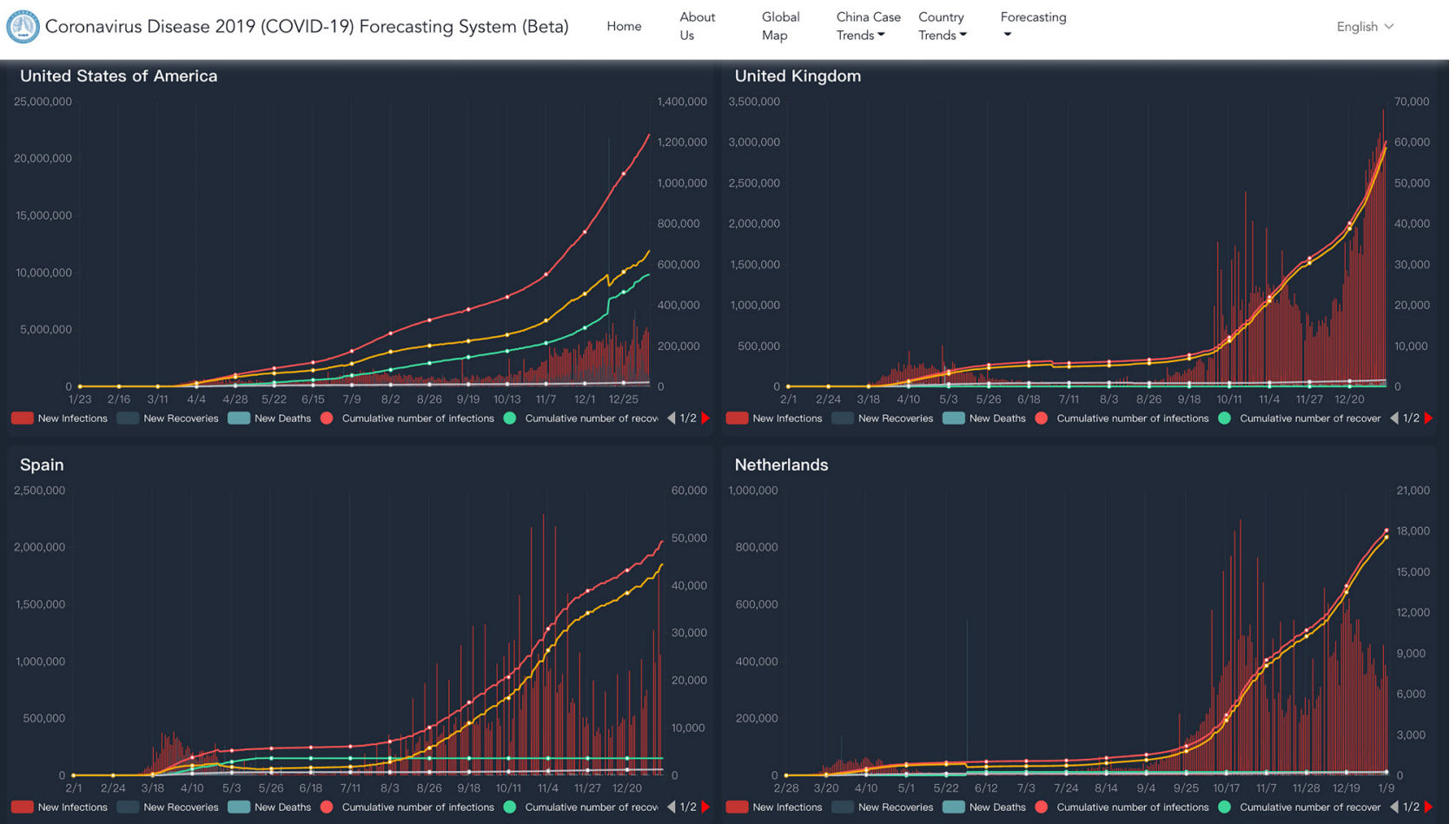

Figure 4 The pandemic information for every country. Red bar, new infections; Black bar, new recoveries; Blue bar, new deaths; Red line, cumulative number of infections; Green line, cumulative number of recoveries; Gray line, cumulative number of deaths; Yellow line, active number of infections. 


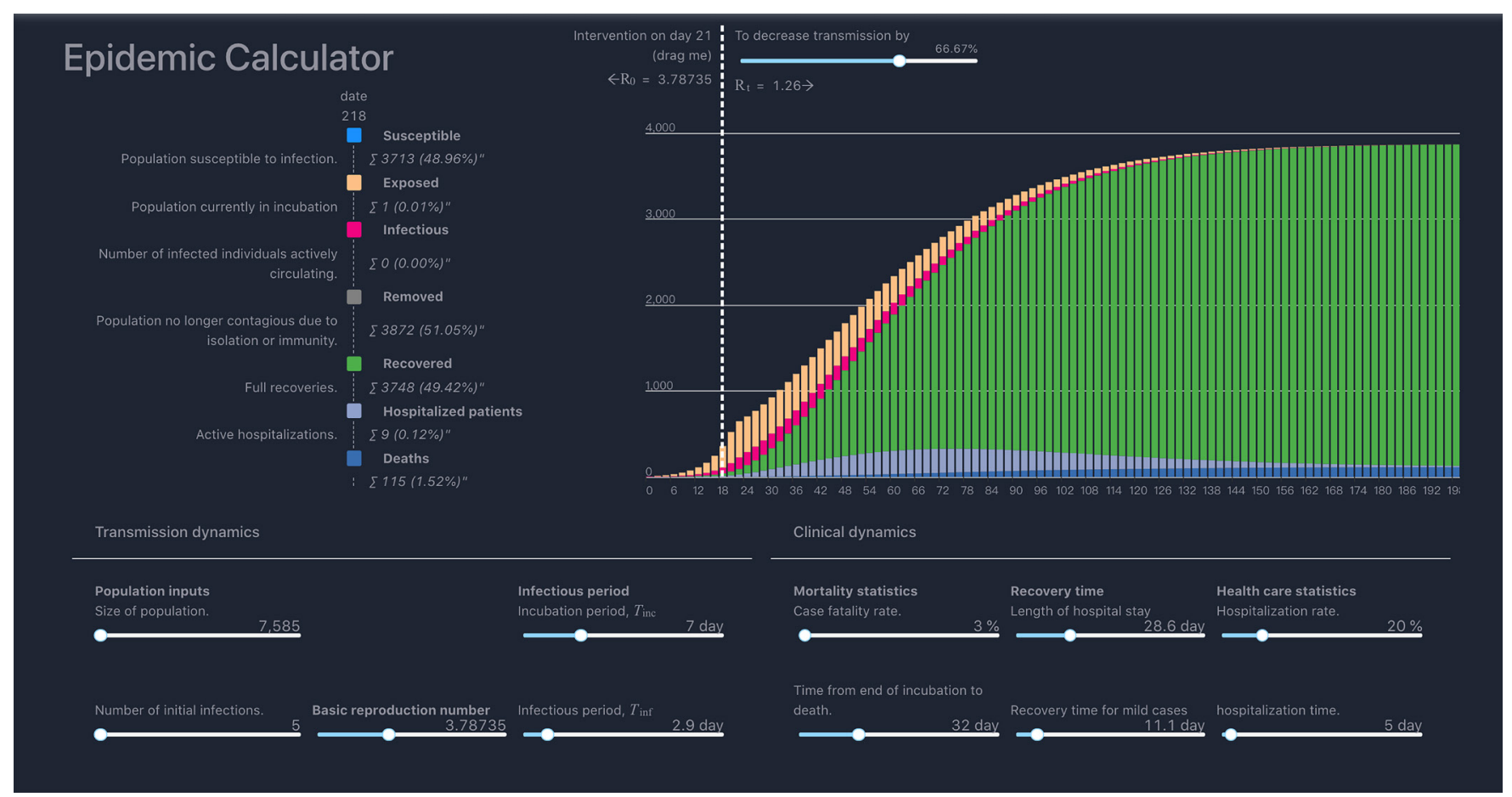

Figure 5 Epidemic Calculator.

cumulative number of confirmed cases is estimated to be approximately 4.5 million (95\% CI: 5,250,175-3,297,076; Figure 6B).

Vaccine progress: Real-time updates of vaccine research and development information, and easy access to the latest progress (Figure 7).

It is hoped that the pandemic prediction platform will provide high-quality services to the public and valuable reference information for the control of SARS-CoV-2 pandemic in China and the world. Only with scientific guidance, the transmission chain of SARS-CoV-2 be cut off as early as possible, which could effectively prevent an outbreak of COVID-19.

The main personnel involved in the development of the system included: Jianxing He, Zifeng Yang, Zhiqi Zeng, Ke Wang, Jundong Tan. The completion of this project was fully supported by the First Affiliated Hospital of Guangzhou Medical University, Guangzhou Medical University, and Hengqin Whale Precision Medical Technology Co., Ltd. Computing power support for the project was provided by Guangdong Qinzhi Institute of Science and Technology Co., Ltd. 
A Coronavirus Disease 2019 (COVID-19) Forecasting System (Beta) Home About Global China Case Country Forecasting

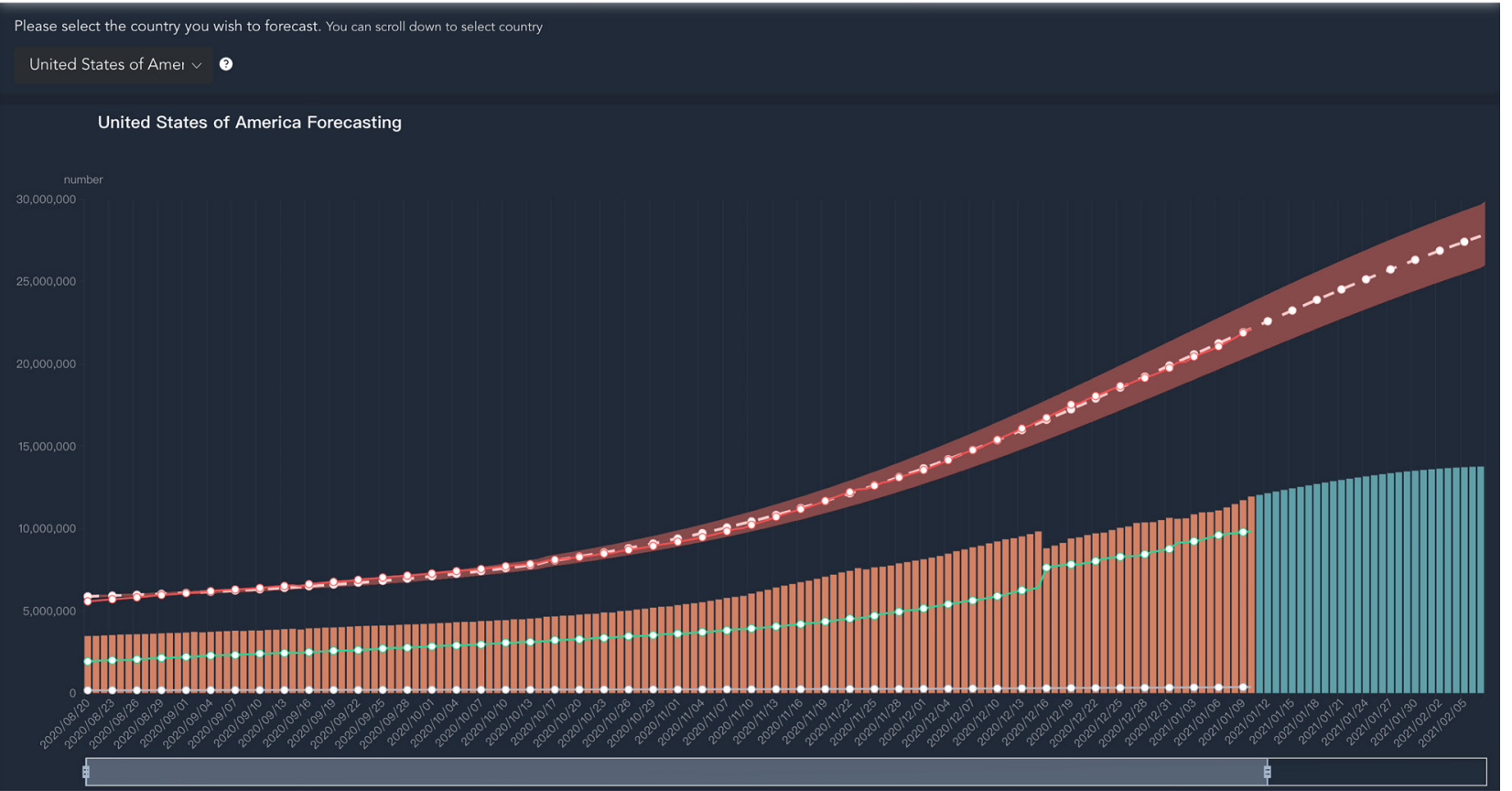

B

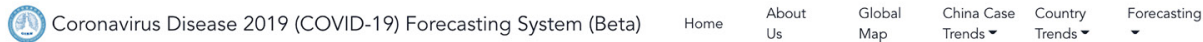

English $\checkmark$

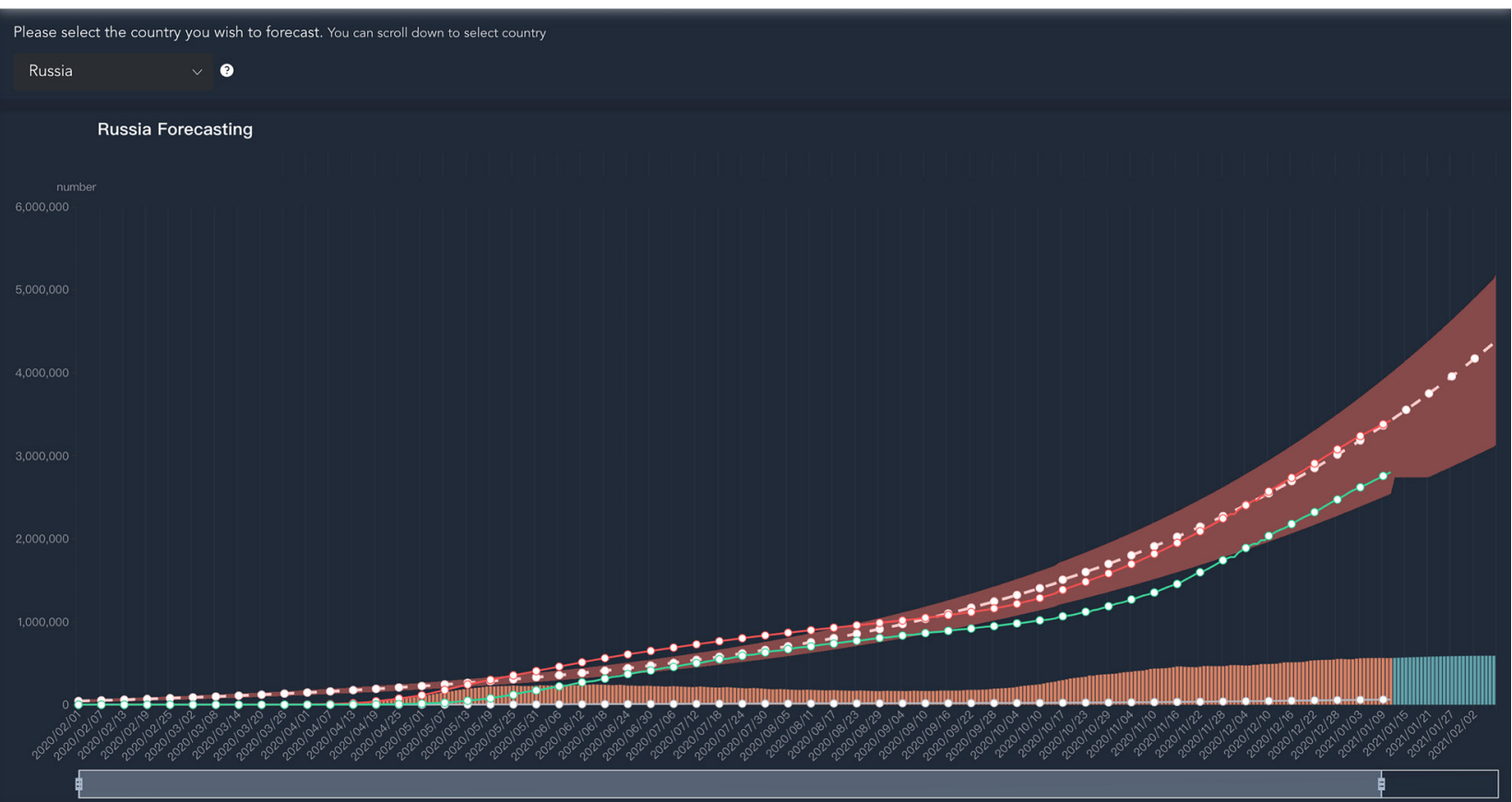

Figure 6 Prediction for America and Russia. (A) America. (B) Russia. Red line, actual number of cumulative infections; Green line, actual number of cumulative recoveries; Gray line, actual number of cumulative deaths; Orange bar, actual number of active infections; Pink line, predicted number of cumulative infections; Blue bar, predicted number of active infections; Red zone, $95 \%$ confidence interval. 


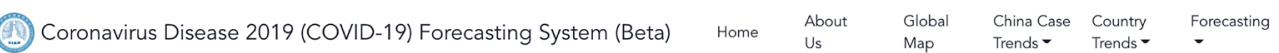

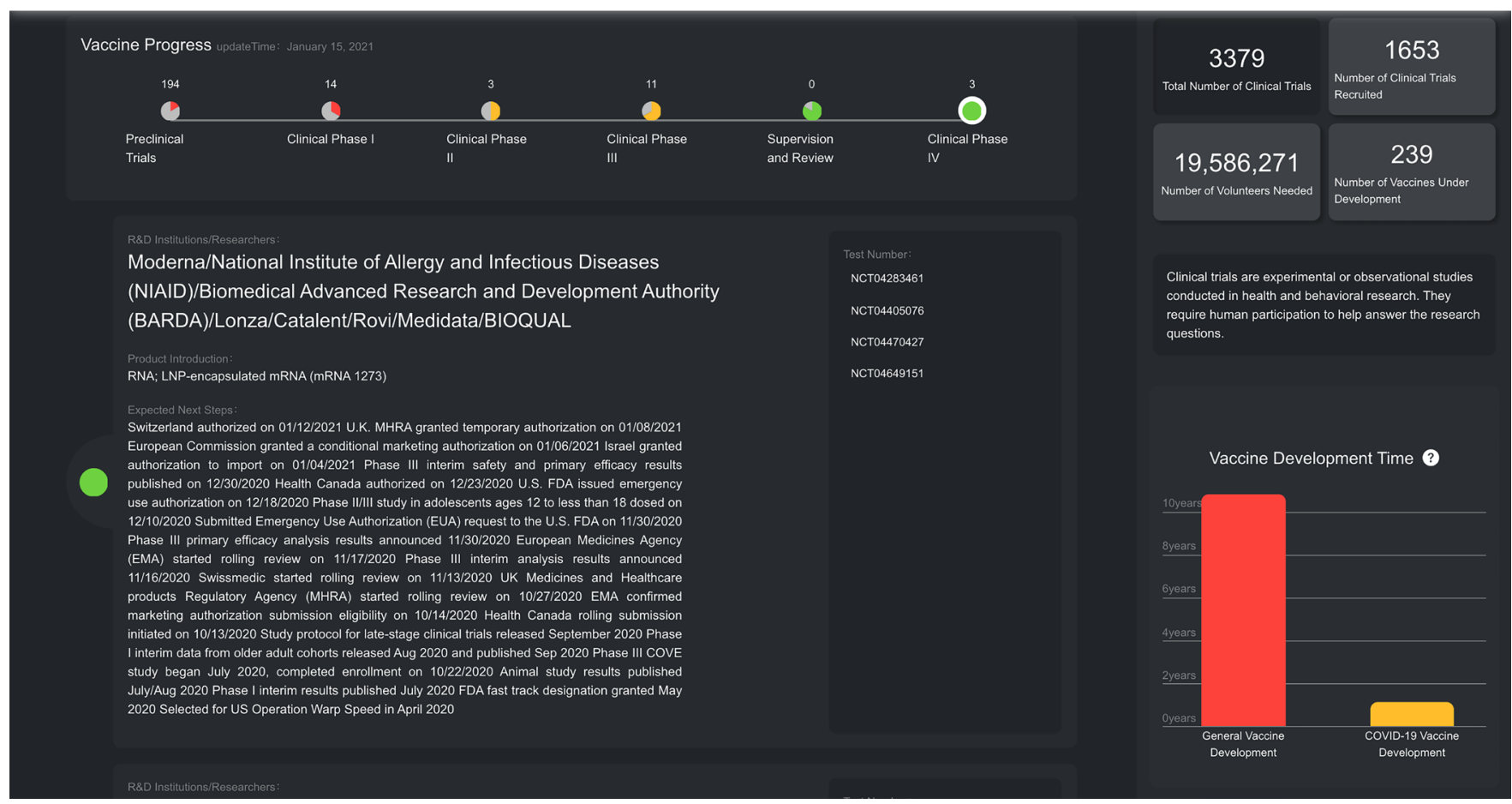

Figure 7 Vaccine progress.

\section{Acknowledgments}

Funding: This work was supported by Guangzhou Institute of Respiratory Health Open Project (Funds provided by China Evergrande Group) - (2020GIRHHMS23).

\section{Footnote}

Provenance and Peer Review: This article was commissioned by the editorial office, Fournal of Thoracic Disease. The article did not undergo external peer review.

Conflicts of Interest: The authors have completed the ICMJE uniform disclosure form (available at http://dx.doi. org/10.21037/jtd-2021-02). Nanshan Zhong serves as Editor-in-Chief of Journal of Thoracic Disease. Jianxing He serves as Executive Editor-in-Chief of Fournal of Thoracic Disease. The other authors have no conflicts of interest to declare.

Ethical Statement: The authors are accountable for all aspects of the work in ensuring that questions related to the accuracy or integrity of any part of the work are appropriately investigated and resolved.

Open Access Statement: This is an Open Access article distributed in accordance with the Creative Commons Attribution-NonCommercial-NoDerivs 4.0 International License (CC BY-NC-ND 4.0), which permits the noncommercial replication and distribution of the article with the strict proviso that no changes or edits are made and the original work is properly cited (including links to both the formal publication through the relevant DOI and the license). See: https://creativecommons.org/licenses/by-nc-nd/4.0/.

Cite this article as: Yang Z, Zeng Z, Wang K, Zhong N, He J. Scientific guidance to fight the pandemic: the Coronavirus Disease 2019 (COVID-19). J Thorac Dis 2021;13(2):505-510. doi: 10.21037/jtd-2021-02 